\title{
BMJ Open The association between vertebrobasilar insufficiency and the risk of dementia: a nationwide register-based retrospective cohort study in Taiwan
}

\author{
Fu-Chi Yang, ${ }^{1}$ Shao-Yuan Chen, ${ }^{2,3,4}$ Jiu-Haw Yin, ${ }^{1,5}$ Chun-Chieh Lin, ${ }^{1}$ \\ Yueh-Feng Sung, ${ }^{1}$ Chung-Hsing Chou, ${ }^{1}$ Chi-Hsiang Chung, ${ }^{6,7,8}$ Wu-Chien Chien, ${ }^{7,8}$ \\ Chia-Kuang Tsai, ${ }^{1}$ Chia-Lin Tsai, ${ }^{1}$ Guan-Yu Lin, ${ }^{1}$ Jiunn-Tay Lee ${ }^{1}$
}

To cite: Yang F-C, Chen S-Y, Yin J-H, et al. The association between vertebrobasilar insufficiency and the risk of dementia: a nationwide registerbased retrospective cohort study in Taiwan. BMJ Open 2017;7:e017001. doi:10.1136/ bmjopen-2017-017001

- Prepublication history and additional material is available. To view please visit the journal (http://dx.doi.org/ 10.1136/ bmjopen-2016-017001).

Received 27 March 2017 Revised 16 May 2017 Accepted 1 June 2017

CrossMark

For numbered affiliations see end of article.

Correspondence to

Dr Jiunn-Tay Lee;

jiunntay@gmail.com

\section{ABSTRACT}

Objectives Neurodegenerative disorders are reportedly characterised by decreased regional cerebral blood flow. However, the association between vertebrobasilar insufficiency (VBI) and dementia remains unclear. In this nationwide, population-based, retrospective cohort study, we explored the potential association between VBI and dementia.

Design Nationwide population-based cohort study. Setting Patients with VBI were newly diagnosed between 2000 and 2005 from the Taiwan National Health Insurance Research Database.

Participants We included 3642 subjects as the VBI group. The control cohort included 14568 randomly selected age-matched and sex-matched VBI-free individuals. Outcome measures All subjects were followed until the diagnosis of dementia, death or the end of 2010. Patients with VBI, dementia (viz, vascular and non-vascular, including Alzheimer's) subtypes and other confounding factors were identified according to the International Classification of Diseases Clinical Modification Codes. Cox proportional hazards regression analysis was employed to examine adjusted HRs after adjusting for confounding factors.

Results Patients with VBI had a 1.807 -fold $(95 \% \mathrm{Cl}$ 1.643 to $1.988, p<0.001$ ) higher risk to develop all-cause dementia than individuals without VBI. The risk was significantly higher in the VBI group than in the non-VBI group regardless of age ( $<65$ years: HR: $2.997,95 \% \mathrm{Cl}$ 1.451 to $6.454, p<0.001 ; \geq 65$ years: HR: $1.752,95 \% \mathrm{Cl}$ 1.584 to $1.937, p<0.001)$. The VBI group had a higher risk of all-cause dementia than the non-VBI group regardless of sex and follow-up time intervals ( $<1$ year, $1-2$ years and $\geq 2$ years).

Conclusion Patients with VBI appear to have an increased risk of developing dementia. Further research is needed to investigate the underlying pathophysiology.

\section{INTRODUCTION}

Vertebrobasilar insufficiency (VBI) mainly affects elderly people and involves reduced blood flow in the posterior part of the brain. The vertebrobasilar (VB) flow system is also
Strengths and limitations of this study

- The major strength of this study is the use of nationwide population-based data, with a large sample size providing sufficient statistical power to explore the relationship between vertebrobasilar insufficiency (VBI) and dementia.

- The longitudinal observational design of this study, with a lengthy follow-up, enabled us to assess the temporal relationship between VBI and dementia.

- The National Health Insurance Database dataset of this study is derived from an administrative coding database, lacking details of clinical symptoms and signs of dementia, such as the predominant symptoms, mental or mood status, imaging data and other laboratory results.

called the posterior circulation, comprising the vertebral arteries, basilar artery, and posterior cerebral arteries and their branches, supplying the brainstem, thalamus, hippocampus, cerebellum, and regions of the occipital and temporal lobes. ${ }^{1}$ VBI typically arises because of the formation of atherosclerotic plaques, resulting in insufficient blood flow through the posterior circulation and is essentially thought to be a transient ischaemic attack (TIA) in the posterior circulation. ${ }^{2}$ VBI can present with transient and recurrent impairments, including dizziness, instability in standing and walking, double vision, hearing loss, speech disorders, nausea or vomiting. ${ }^{34}$ Previous studies have indicated that recurrent VBI may increase the future risk of posterior circulation stroke. ${ }^{56}$ Furthermore, some patients with VBI may suffer from progressive deterioration of memory and higher cortical functions, as well as dementia. $^{78}$

Alzheimer's disease (AD) is one of the most common neurodegenerative diseases, 
characterised by slowly progressive deterioration of memory and cognitive function, and decreased regional cerebral blood flow. ${ }^{9}{ }^{10}$ Dementia can be classified as non-vascular dementia (non-VD) (including $\mathrm{AD}$ ), accounting for $80 \%-90 \%$ of all cases of dementia, and vascular dementia (VD), accounting for the remainder. ${ }^{11}$ The risk factors of dementia increase with age and include a variety of other conditions, such as diabetes, hypertension, dyslipidaemia, ischaemic heart disease, alcoholism, heart failure, depression, head injury, stroke and Parkinson's disease. ${ }^{12-15}$ Furthermore, vascular-associated cognitive decline has been reported to be more common than previously thought. ${ }^{16}$ Investigating links between these risk factors and dementia may therefore provide appropriate preventative strategies, and reduce the risk and socioeconomic burden of dementia.

Patients with carotid stenosis (anterior circulation) are more susceptible to cognitive and memory impairment. ${ }^{1718}$ However, the association between VBI (posterior circulation) and cognitive impairment has been poorly studied. Patients with VBI perform below average on a number of cognitive tests with age-corrected scores ${ }^{19}$ and have significantly lower Benton Facial Recognition Test scores than age-matched controls, which could indicate early changes in visuospatial cognitive skills. ${ }^{20}$ Moreover, 3 months of treatment with a cerebral vasodilator resulted in cognitive improvements in patients with VBI. ${ }^{21}$ These studies suggest that certain cognitive impairments exist in patients with VBI.

Taken together, the association between VBI and dementia remains to be determined, and the temporal relationship between VBI and dementia is still unknown. Therefore, we designed a nationwide, population-based cohort study aimed at exploring the possible temporal association between VBI and dementia.

\section{METHODS}

\section{Ethics statement}

This study was approved by the joint institutional review board of Tri-Service General Hospital (approval TSGHIRB No. 2-104-05-045). Confidentiality was assured following the data regulations of the Health and Welfare Data Science Center, Ministry of Health and Welfare, Executive Yuan, Taiwan, which releases patients' data to investigators only for research to protect patients' privacy. The study group and a control cohort were selected from the Longitudinal Health Insurance Database (LHID). Because data in the LHID is de-identified, the requirement for obtaining informed consent from the study participants was waived by the joint institutional review board. All the study methods were according to the guidelines approved by the joint institutional review board and forenamed governmental regulations.

\section{Data source}

This study was conducted using claims data from the National Health Insurance Research Database (NHIRD), which is maintained by the National Health Research Institute (NHRI) in Taiwan. The National Health Insurance (NHI) programme of Taiwan is a universal insurance programme established in 1995, and it is a mandatory single-payer healthcare system, covering the healthcare costs of more than 99\% of Taiwan's residents (approximately 23 million). In this study, we used the LHID in Taiwan (2000-2005), which is a subset of the NHIRD. The LHID contains data of 1 million people, randomly sampled from the NHIRD, and has been demonstrated to be representative the entire population of Taiwan. The LHID contains the demographic data, dates of clinical visits, drug prescriptions and disease diagnosis codes in the format of the International Classification of Disease, Ninth Revision, Clinical Modification (ICD-9-CM). The NHRI reported no statistically significant differences in the sex and age distribution between the LHID subset and the total NHIRD. The NHI has a rigorous monitoring system, whereby it audits healthcare records to validate medical claims for diagnosis and treatment, to ensure that claims for reimbursement are based on valid diagnoses.

\section{Study design and sampled participants}

This population-based cohort study aimed to investigate the association of VBI and the risk of dementia by comparing two groups: the VBI group and the non-VBI group. We identified patients who were 20 years of age and older, with newly diagnosed VBI (ICD-9-CM 433.0, 433.2, 433.3, 435.0, 435.1, 435.3) based on outpatient care data in the NHIRD between 1 January 2000 and 31 December 2005, as the VBI group. The date of diagnosis of VBI was considered as the index date. All diagnoses of VBI were made on the basis of the clinical symptoms and signs with a focus on the cardiovascular and neurological systems and/or supporting imaging studies (Doppler ultrasonography, magnetic resonance angiography or CT angiography) from outpatient departments of participating hospitals and clinics. ${ }^{22}$ For each patient with VBI, four insured subjects were selected randomly from subjects without a diagnosis of VBI in the LHID, as the non-VBI group, and were frequency-matched by sex, age and comorbidities that are known to be dementia risk factors and that were established before the index date. Selection according to comorbidities was based on outpatient data of the following ICD codes: hypertension (ICD-9-CM codes 401-405), diabetes (ICD-9-CM code 250), ischaemic heart disease (ICD-9-CM codes 410-414), hyperlipidaemia (ICD-9-CM code 272), tobacco use disorder (ICD-9-CM code 305.1), alcoholism (ICD-9-CM codes 291, 303.9, 334.4, 980.0), obesity (ICD-9-CM codes 278, 649.1, 783.1), atrial fibrillation (ICD-9-CM code 427.3), Parkinson's disease (ICD-9-CM code 332.0), cerebral vascular accident (ICD-9-CM codes 430-432, 433-437), major depression (ICD-9-CM code 296), chronic kidney disease (ICD-9-CM code 585) and carotid artery insufficiency (ICD-9-CM code 433.1, 435.8). Each patient in both groups was then followed up from the 
Outpatient events of Longitudinal Health Insurance Database (LHID) in 2000-2005 in Taiwan

73682099 events; 967854 individuals

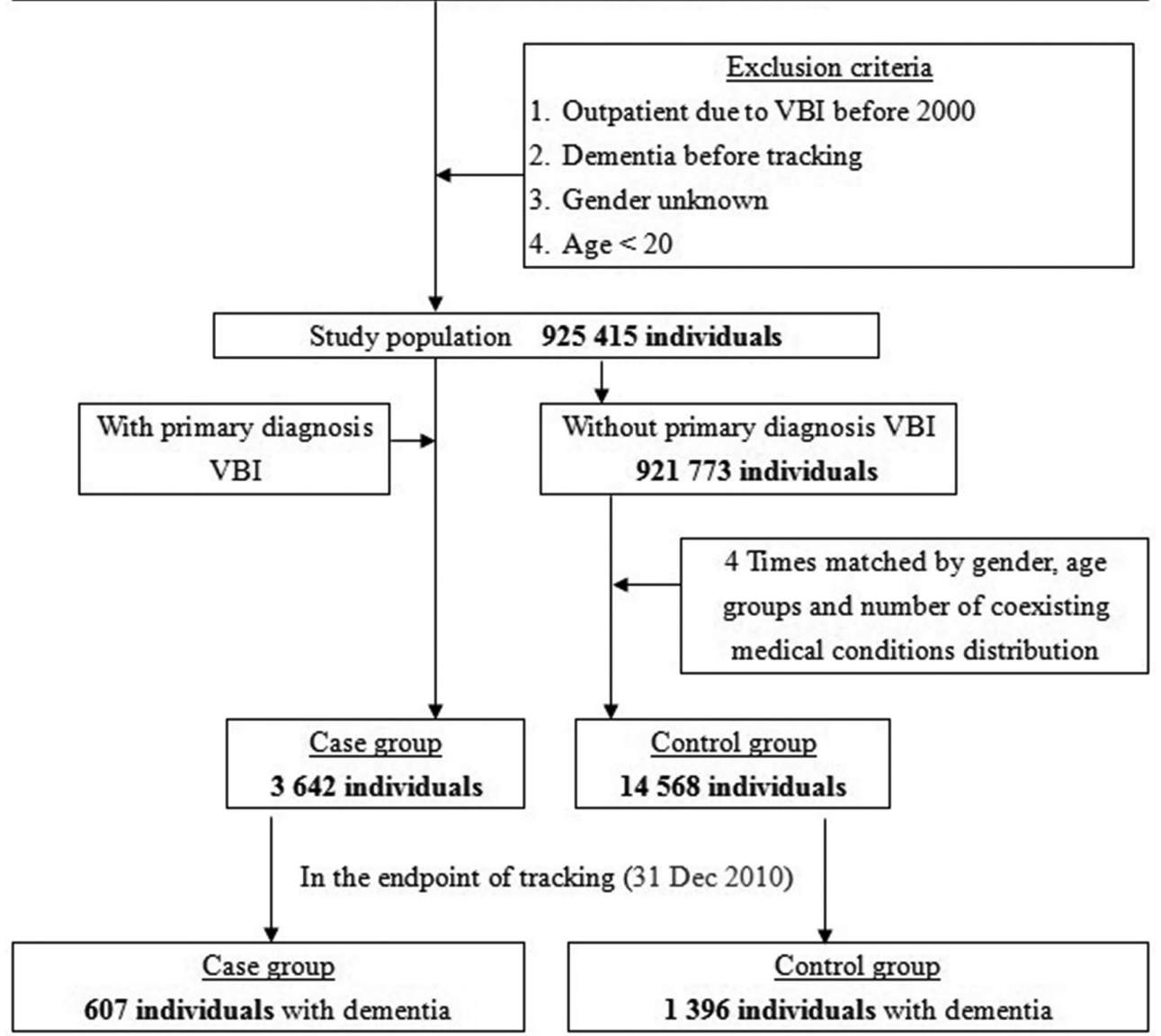

Figure 1 Flowchart of study sample selection from the National Health Insurance Research Database in Taiwan.

index date until the occurrence of dementia. For those who did not have dementia, the last day of follow-up was defined as the date of insurance withdrawal or the last day of the study period (31 December 2010) (figure 1).

\section{Outcome measures and definitions of dementia subtypes by ICD classification}

The primary outcome was classified as all-cause dementia (ICD-9-CM 290-294 and 331.0). The diagnosis of dementia was made based on the diagnostic criteria of the Diagnostic and Statistical Manual of Mental Disorders, Fourth Edition, ${ }^{23}$ after assessing the patients' medical history, daily activities, physical and social function, behaviour and cognitive function, blood tests and brain imaging. We also examined different subtypes of dementia. VD was identified in the claims data using the ICD-9-CM code 290.4; all of the remaining patients with dementia were classified as non-VD, and patients with $\mathrm{AD}$ were differentiated from those with non-VD using ICD-9-CM code 331.0.

\section{Statistical analyses}

All analyses were performed using SPSS V.22 software (SPSS). Continuous variables were presented as mean \pm SD and categorical variables as frequencies and percentages. Differences between the study and control groups in terms of demographic characteristics (age and sex) and comorbidities (hypertension, diabetes, ischaemic heart disease, hyperlipidaemia, smoking, alcoholism, obesity, atrial fibrillation, Parkinson's disease, cerebrovascular accident, major depression, chronic kidney disease and carotid artery insufficiency) were examined by $\chi^{2}$ or Fisher's exact test. Multivariate Cox proportional hazards regression analysis was used to determine the risk of dementia, and the results were presented as HRs with $95 \%$ CIs. To investigate the interaction of covariates with the association between VBI and dementia, we also calculated adjusted HRs, stratified by age $(<65$ years, $\geq 65$ years), sex and follow-up time. A two-tailed $p$ value $<0.05$ was considered to indicate statistical significance. Although dementia mostly affects older people, those aged $\geq 65$ years, it can occur in people aged $<65$ years, known as young-onset dementia. ${ }^{24}$ Therefore, we performed analysis of age as a two-level ordinal variable (with cut-offs at 65 years). To explore whether VBI is a time-dependent risk factor for dementia in this study, we also investigated the association between follow-up time after diagnosis of VBI and the risk of dementia. Since patients with VBI may be more likely to die from stroke or other related medical conditions, we further 
Table 1 Baseline demographic status and comorbidity compared between comparison and VBI group

\begin{tabular}{lccc}
\hline Variable & $\begin{array}{l}\text { VBI group } \\
\mathbf{n}=\mathbf{3 6 4 2}(\%)\end{array}$ & $\begin{array}{l}\text { Comparison group } \\
\mathbf{n}=\mathbf{1 4 5 6 8}(\%)\end{array}$ & $\mathbf{p}$ Value \\
\hline Age, years (SD)* & $67.37(13.82)$ & $65.41(15.99)$ & 0.733 \\
$\quad<65$ & $1362(37.40)$ & $5.448(37.40)$ & 0.999 \\
\hline Sex & $2280(62.60)$ & $9120(62.60)$ & \\
$\quad$ Female & & & 0.153 \\
$\quad$ Male & $1794(49.26)$ & $7176(49.26)$ & 0.298 \\
Comorbidity & $1848(50.74)$ & $7392(50.74)$ & 0.742 \\
\hline Hypertension & & & 0.109 \\
DM & $671(18.42)$ & $2443(16.77)$ & 0.436 \\
IHD & $304(8.35)$ & $1105(7.59)$ & 0.800 \\
\hline Hyperlipidaemia & $204(5.60)$ & $803(5.51)$ & - \\
\hline TUD & $31(0.85)$ & $94(0.65)$ & 0.276 \\
Alcoholism & $1(0.03)$ & $8(0.05)$ & 0.146 \\
Obesity & $0(0)$ & $1(0.01)$ & 0.178 \\
AF & $0(0)$ & $0(0)$ & 0.298 \\
\hline PD & $27(0.74)$ & $126(0.86)$ & - \\
CVA & $28(0.77)$ & $87(0.60)$ & 0.072 \\
\hline Depression & $106(2.91)$ & $471(3.23)$ & $16(0.11)$ \\
CKD & $7(0.19)$ & $0(0)$ & $36(0.25)$ \\
CAI & $0(0)$ & & \\
\hline At & $7(0.19)$ & & \\
\hline
\end{tabular}

*t-test.

AF, atrial fibrillation; CAI, carotid artery insufficiency; CKD, chronic kidney disease; CVA, cerebral vascular accident; DM, diabetes mellitus; $I H D$, ischaemic heart disease; PD, Parkinson's disease; TUD, tobacco use disorder; VBI, vertebrobasilar insufficiency.

assessed adjusted HRs of death and utilised competing risk models as well as Cox models.

\section{RESULTS}

For the period 2000-2005, this study identified 3642 patients newly diagnosed with VBI and 14568 subjects without VBI. There were no significant differences between the VBI group and non-VBI group in the distribution of sex, age and comorbidities (table 1).

During an average follow-up of $4.23 \pm 1.57$ years, a total of 607 patients with VBI (16.7\%) of the VBI group and 1396 non-VBI individuals $(9.6 \%)$ of the non-VBI group developed dementia. Patients with VBI had a 1.807 -fold (95\% CI 1.643 to $1.988, \mathrm{p}<0.001$ ) higher risk of developing all-cause dementia than individuals without VBI (table 2).

To explore whether VBI is an age-dependent risk factor for all-cause dementia, patients were divided into two groups according to age, namely $<65$ and $\geq 65$ years. In comparison with age-matched/sex-matched controls, the VBI group had a higher risk of all-cause dementia than the non-VBI group, regardless of age $(<65$ years: HR: 2.997, $95 \%$ CI 1.451 to $6.454, \mathrm{p}<0.001$; $\geq 65$ years: HR: $1.752,95 \%$ CI 1.584 to $1.937, \mathrm{p}<0.001)$. We also examined whether VBI is a sex-dependent risk factor for development of dementia. Cox regression analysis revealed that the VBI group had a higher risk of all-cause dementia, regardless of sex (men: HR: 1.732, 95\% CI 1.514 to 1.981 , p<0.001; women: HR: 1.888 , 95\% CI 1.649 to 2.160 , $\mathrm{p}<0.001$ ) (table 2). Since age has a potential impact on dementia occurrence, we also examined the associations between VBI and the risk of dementia after controlling for age as a continuous variable. The results were similar, as shown in online supplementary table S1.

We next analysed the incidence of dementia using multivariate Cox proportional hazards regression analysis, based on follow-up time intervals. Our data showed that patients with VBI were likely to develop subsequent dementia after diagnosis regardless of follow-up time intervals $(<1$ year, 1-2 years and $\geq 2$ years) (table 3 ). Kaplan-Meier analysis showed that, compared with the matched controls, patients with VBI had a significantly higher incidence of all-cause dementia (log-rank test $\mathrm{p}<0.001$ ) (figure 2$)$. We further examined the associations between VBI and the risk of different subtypes of dementia, as shown in online supplementary tables S2 and S3.

After considering the competing risk of death, the results were similar. Patients with VBI had a 1.725 -fold $(95 \%$ CI 1.452 to $1.996, \mathrm{p}<0.001)$ higher risk of developing all-cause dementia than individuals without VBI (see online supplementary figure S1). The risk was most 
Table 2 Incidence of dementia and hazard ratios (multivariate Cox proportional hazards regression analysis) for the study cohort according to stratification by age and sex

\begin{tabular}{|c|c|c|c|c|c|c|c|c|}
\hline \multirow[b]{2}{*}{ Variable } & \multicolumn{3}{|c|}{ VBI group } & \multicolumn{3}{|c|}{ Non-VBI group } & \multirow[b]{2}{*}{ Crude HR (95\% CI) } & \multirow[b]{2}{*}{ Adjusted HR $(95 \% \mathrm{Cl})$} \\
\hline & Event & PYs & Rate & Event & PYs & Rate & & \\
\hline All dementia & 607 & 14802 & 41.01 & 1396 & 62194 & 22.45 & 2.285 (2.179 to 2.397$)^{* \star *}$ & $1.807(1.643 \text { to } 1.988)^{*+*}$ \\
\hline$<65$ & 72 & 6388 & 11.27 & 97 & 25961 & 3.74 & $3.012(1.745 \text { to } 5.642)^{\star \star *}$ & $2.997(1.451 \text { to } 6.454)^{* \star}$ \\
\hline$\geqq 65$ & 535 & 8415 & 63.58 & 1299 & 36234 & 35.85 & $2.250(2.140 \text { to } 2.366)^{\star \star \star}$ & $1.752(1.584 \text { to } 1.937)^{\star \star \star}$ \\
\hline Male & 300 & 7058 & 42.51 & 721 & 29709 & 24.27 & $2.337(2.092 \text { to } 2.393)^{\star \star \star}$ & $1.732(1.514 \text { to } 1.981)^{\text {*k }}$ \\
\hline Female & 307 & 7745 & 39.64 & 675 & 32485 & 20.78 & $2.336(2.183 \text { to } 2.498)^{* * *}$ & $1.888(1.649 \text { to } 2.160)^{* * x}$ \\
\hline
\end{tabular}

Model adjusted for age, sex, hypertension, diabetes mellitus, ischaemic heart disease, hyperlipidaemia, tobacco use disorder, alcoholism, obesity, atrial fibrillation, Parkinson's disease, cerebral vascular accident, depression, chronic kidney disease and carotid artery insufficiency; all variables in the model were significant in univariate analysis. Interaction: VBI $\times$ age groups: $p=0.025^{*}, \mathrm{VBI} \times$ sex: $p=0.679$.

${ }^{*} p<0.05$.

${ }^{* * *} \mathrm{p}<0.001$.

PYs, person-years; Rate, incidence per 10000 person-years; VBI, vertebrobasilar insufficiency.

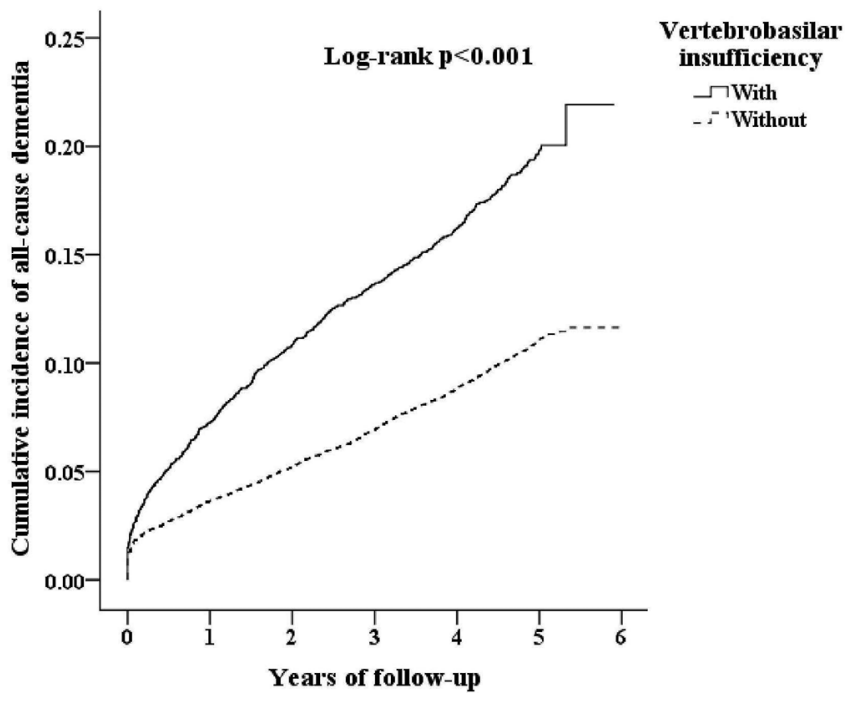

Figure 2 Cumulative incidence curves of all-cause dementia for the individual with and without vertebrobasilar insufficiency.

prominent in both the <65year group (HR: 2.845, 95\% CI 1.376 to $5.897, \mathrm{p}<0.01)$ and the $\geq 65$ year group (HR: $1.672,95 \%$ CI 1.434 to $1.963, \mathrm{p}<0.001)$ in the VBI group, as compared with the non-VBI group (see online supplementary table S4). The VBI group had a higher risk of all-cause dementia than the non-VBI group regardless of sex and follow-up time intervals ( $<1$ year, $1-2$ years and $\geq 2$ years) (see online supplementary table S5).

\section{DISCUSSION}

To the best of our knowledge, this is the first longitudinal study using a nationwide, population-based database to demonstrate the increased risk of all-cause dementia in patients with VBI. Our findings showed that patients with VBI had a 1.807-fold higher risk of developing all-cause dementia in comparison with a matched non-VBI cohort. The risk of developing subsequent dementia was higher in the VBI group, regardless of age, when compared with the non-VBI group. Furthermore, the impact of VBI on dementia risk was independent of sex and various follow-up intervals.

The present results support those of previous studies reporting an association between VBI and cognitive impairment. ${ }^{19} 20$ Similarly, patients with $\mathrm{AD}$ have been found to show reduced cerebral blood flow in the

Table 3 Incidence of dementia and HRs (multivariate Cox proportional hazards regression analysis) for the study cohort according to various follow-up periods

\begin{tabular}{|c|c|c|c|c|c|c|c|c|}
\hline \multirow[b]{2}{*}{ Follow-up } & \multicolumn{3}{|c|}{ VBI group } & \multicolumn{3}{|c|}{ Non-VBI group } & \multirow[b]{2}{*}{ Crude HR $(95 \% \mathrm{Cl})$} & \multirow[b]{2}{*}{ Adjusted HR (95\% Cl) } \\
\hline & Event & PYs & Rate & Event & PYs & Rate & & \\
\hline$<1$ year & 248 & 118 & 2100.27 & 508 & 296 & 1713.44 & $1.958(1.814 \text { to } 2.112)^{* t * t}$ & $1.326(1.139 \text { to } 1.544)^{* *}$ \\
\hline$\geq 2$ years & 247 & 14398 & 17.16 & 682 & 61027 & 11.18 & $2.108(1.960 \text { to } 2.267)^{\star * \star}$ & $1.537(1.329 \text { to } 1.778)^{\star \star}$ \\
\hline
\end{tabular}

Model adjusted for age, sex, hypertension, diabetes mellitus, ischaemic heart disease, hyperlipidaemia, tobacco use disorder, alcoholism, obesity, atrial fibrillation, Parkinson's disease, cerebral vascular accident, depression, chronic kidney disease and carotid artery insufficiency; all variables in the model were significant in univariate analysis. Interaction: VBI $\times$ follow-up groups: $\mathrm{p}=0.726$.

PYs, person-years; Rate, incidence per 10000 person-years; VBI, vertebrobasilar insufficiency.

${ }^{* * *} p<0.001$. 
posterior circulation, ${ }^{25}$ and patients with lower regional cerebral blood flow in the vertebrobasilar system have been found to show verbal memory impairment, language disturbances and apathy. ${ }^{26}$ Cervicocerebral stenosis has also been shown to be associated with cognitive dysfunction, and revascularisation of the anterior and posterior circulation using stents has been reported to improve cognition. ${ }^{27-29}$ Collectively, these studies, along with our own, reinforce the association between decreased regional cerebral blood flow, especially in the posterior circulation, and cognitive decline. Our study further considers the potential medical and psychological confounding factors and comorbidities, using a large nationwide sample size and a longitudinal design, providing results that are more generalisable to the general population.

$\mathrm{AD}$ is considered to be the most common cause of dementia, followed by VD. ${ }^{11}{ }^{16}$ We demonstrated that, in addition to $\mathrm{AD}$, patients with VBI also exhibit a higher risk of VD than a control cohort. This is in agreement with a previous study that identified cerebrovascular insufficiency as an important risk factor for incident dementia. ${ }^{30}$ Furthermore, poor cognitive performance has been reported to be strongly associated with stroke risk factors and cerebral microvascular disease. ${ }^{31} 32$ The vascular risk factors for VBI, for example, hypertension, hyperlipidaemia, diabetes and obesity, are also reported to be independent mid-life risk factors for clinical AD. ${ }^{33} 34$ Therefore, in this study, after considering the possible vascular risk factors or comorbidities of dementia, our multivariate Cox analyses demonstrated that VBI remains an independent predictor of subsequent dementia risk.

Dementia is generally acknowledged to be an ageing-related disorder, and its incidence is known to increase with age in the general population. ${ }^{35}$ Ageing is also thought to be associated with subcortical microangiopathy, and elderly people may have impaired cerebral blood flow to the brain's posterior regions. ${ }^{10}$ We demonstrated that VBI is more likely to result in dementia regardless of age. Previous studies have reported a slightly greater probability of development of dementia among women. ${ }^{36}$ However, we found that the higher risk of dementia in the VBI group was not influenced by the patient's sex after adjusting for multivariate effects. This result partly supports a previous report that demonstrated that the severity of cognitive impairment was independent of sex in patients with stroke in the posterior cerebral artery territory. ${ }^{37}$ In addition, in our results, the higher risk of dementia in the VBI group was not influenced by varying follow-up time intervals. Collectively, we suggest that these findings indicate that VBI is an independent potential risk factor for dementia regardless of age, sex and follow-up time.

Several mechanisms may underlie the pathological association between VBI and dementia. First, amnesia and memory impairment are thought to be later complications of VBI, and previous studies have indicated that this is due to hypoperfusion of the terminal branches of the vertebrobasilar system, which supply areas of the brain, such as the thalamus, hippocampus, occipital lobe and cerebellum, which are involved in important cognitive functions, particularly memory, visual-spatial functioning and visuomotor adaptations. ${ }^{8}{ }^{39}$ Papez circuit hypoperfusion or disruption at the mammillo-thalamic tract and possibly thalamo-medial-temporal disconnection have also been proposed to be responsible for memory impairment. ${ }^{26}$ Second, deposition of amyloid, which is a protein normally found in the brain, has been known to play a role in $\mathrm{AD}$ pathogenesis. Cerebral amyloid angiopathy has also been considered to be associated with vascular cognitive impairment and may exist as a feature of $\mathrm{AD}{ }^{40}$ Moreover, increasing evidence from experimental animal models has suggested an association between the vascular effects of amyloidopathy and cognitive impairment. ${ }^{41}$ Finally, advanced neuroimaging studies have reported that patients with carotid stenosis have extensive structural and functional disruptions in brain networks that correlate with neurocognitive decline, even at a subclinical stage ${ }^{4243}$ These findings suggest that carotid stenosis may not only affect focal neural circuits but also disrupt remote interhemispheric connections that are related to cognition and memory. Since these studies support the relationship between neuropsychological performance and functional and structural disruptions in carotid stenosis (anterior circulation), it may reasonably be hypothesised that such brain structural and functional changes in relation to cognitive and memory changes may also exist in patients with VBI (with an affected posterior circulation). Further studies are warranted to assess this hypothesis.

The major strength of the present study is the use of a large sample size (NHIRD), which provided sufficient statistical power to explore the relationship between VBI and dementia. However, the present study also had certain limitations. First, the diagnosis of VBI and dementia was based on diagnostic codes entered into the health insurance system database by the physicians; therefore, additional clinical information about VBI or dementia (imaging data, laboratory and cognitive test data, or prognoses of both diseases) was not available. Thus, we could not determine whether the degree of VBI was associated with dementia severity and other dementia characteristics. Furthermore, the inter-rater variability of the investigators may also exist in this study. In the future, protocols for training the site personnel to make a more correct diagnosis of VBI and dementia, and to decrease the inter-rater variability, are needed. Second, in this study, the NHIRD dataset was derived from an administrative coding database lacking details of clinical symptoms and signs of dementia, such as the predominant symptoms, mental or mood status, imaging data and other laboratory results. Thus, the precise diagnosis of other subtypes of the dementia was not available in this study, and the results regarding the subtypes of the dementia (see online supplementary tables 2-5) should be interpreted with caution. Third, as a retrospective longitudinal study, we had no access to information about some adaptable or 
personal potential confounders, such as education, lifestyle (smoking or alcohol consumption), physical activity, body mass index, social engagement, family histories or medications, which were not available in the NHIRD and which may potentially confound our results. Fourth, in this study, the comorbidities were matched when choosing controls for dementia cases. Although this matching strategy may have advantages in eliminating the potential influence of confounders, it may be vulnerable to overmatching, especially when the matching variable has some relationship with the outcome. For example, some of the comorbidities might also involve the pathways linking VBI to dementia, which may mask the association between the exposure and outcome. Finally, in this study, we used a longitudinal observational design, with a long follow-up period, which enabled us to assess the temporal relationship between VBI and dementia; however, our findings demonstrated only an association, but not necessarily a causal relationship. Further study is warranted to examine the potential causal relationship between VBI and dementia. Collectively, because of the underlying methodological limitations in this study mentioned above, the present findings in this study should be interpreted and generalised with caution.

In conclusion, in this large-scale, nationwide, population-based longitudinal study, we provided evidence supporting a temporal association between VBI and the risk of dementia regardless of the subjects' age, sex and follow-up intervals. Although the exact mechanism underlying this association is unknown, these findings may provide further insight into a possible shared pathophysiology between VBI and dementia. Additional studies are warranted to elucidate the mechanism and causality of this relationship.

\section{Author affiliations}

${ }^{1}$ Department of Neurology, Tri-Service General Hospital, National Defense Medical Center, Taipei, Taiwan

${ }^{2}$ Department of Neurology, Cardinal Tien Hospital, New Taipei City, Taiwan ${ }^{3}$ Department of Hyperbaric Medicine, Cardinal Tien Hospital, New Taipei City, Taiwan ${ }^{4}$ School of Medicine, Fu-Jen Catholic University, New Taipei City, Taiwan ${ }^{5}$ Division of Neurology, Department of Medicine, Cheng Hsin General Hospital, Taipei, Taiwan

${ }^{6}$ Taiwanese Injury Prevention and Safety Promotion Association, Taipei, Taiwan ${ }^{7}$ School of Public Health, National Defense Medical Center, Taipei, Taiwan ${ }^{8}$ Department of Medical Research, Tri-Service General Hospital, National Defense Medical Center, Taipei, Taiwan

Contributors Study concept/design: F-CY, S-YC, J-HY, J-TL; data collection and analysis: F-CY, S-YC, J-HY, J-TL, C-CL, Y-FS, C-H Chou, C-H Chung, W-CC, C-KT, C-LT, G-YL; data interpretation: F-CY, S-YC, J-HY, J-TL, C-CL, Y-FS, C-H Chou, C-H Chung, W-CC, C-KT, C-LT, G-YL; manuscript writing: F-CY, J-TL.

Funding This study was supported in part by grants from the Tri-Service General Hospital (TSGH-C104-084;TSGH-C105-084; TSGH-C100-101; TSGH-C101-080; TSGH-C103-085; TSGH-C104-083;TSGH-C105-085; TSGH-C101-159;TSGH-C106-068), Ministry of Science and Technology (MOST1042314-B-016-017-MY3;MOST105-2314-B-016-004-) and the Teh-Tzer Study Group for Human Medical Research Foundation (A1031031). The funders had no role in the study design, data collection and analysis, decision to publish or preparation of the manuscript.

Competing interests None declared.

Patient consent Obtained.
Ethics approval This study was approved by joint institutional review board of Tri-Service General Hospital (approval TSGHIRB No. 2-104-05-045).

Provenance and peer review Not commissioned; externally peer reviewed.

Data sharing statement No additional data are available.

Open Access This is an Open Access article distributed in accordance with the Creative Commons Attribution Non Commercial (CC BY-NC 4.0) license, which permits others to distribute, remix, adapt, build upon this work non-commercially, and license their derivative works on different terms, provided the original work is properly cited and the use is non-commercial. See: http://creativecommons.org/ licenses/by-nc/4.0/

(C) Article author(s) (or their employer(s) unless otherwise stated in the text of the article) 2017. All rights reserved. No commercial use is permitted unless otherwise expressly granted.

\section{REFERENCES}

1. Yamamoto $\mathrm{Y}$, Georgiadis AL, Chang HM, et al. Posterior cerebral artery territory infarcts in the New England Medical Center Posterior Circulation registry. Arch Neurol 1999;56:824-32.

2. Caplan L. Posterior circulation ischemia: then, now, and tomorrow. The Thomas Willis Lecture-2000. Stroke 2000;31:2011-23.

3. Jenkins JS, White CJ, Ramee SR, et al. Vertebral insufficiency: when to intervene and how? Curr Interv Cardiol Rep 2000;2:91-4.

4. Doss A, Phatouros CC. Vertebrobasilar insufficiency. Curr Treat Options Cardiovasc Med 2006;8:111-9.

5. Gulli G, Khan S, Markus HS. Vertebrobasilar stenosis predicts high early recurrent stroke risk in posterior circulation stroke and TIA. Stroke 2009;40:2732-7.

6. Caplan LR, Wityk RJ, Glass TA, et al. New England Medical Center Posterior Circulation registry. Ann Neurol 2004;56:389-98.

7. Mölsä PK, Marttila RJ, Rinne UK. Survival and cause of death in Alzheimer's disease and multi-infarct dementia. Acta Neurol Scand 1986;74:103-7.

8. Rivera VM, Meyer JS, Baer PE, et al. Vertebrobasilar arterial insufficiency with dementia. Controlled trials of treatment with betahistine hydrochloride. J Am Geriatr Soc 1974:22:397-406.

9. Caselli RJ. Current issues in the diagnosis and management of dementia. Semin Neurol 2003;23:231-40.

10. Mazza M, Marano G, Traversi G, et al. Primary cerebral blood flow deficiency and Alzheimer's disease: shadows and lights. J Alzheimers Dis 2011;23:375-89.

11. Morris JC. Dementia update 2003. Alzheimer Dis Assoc Disord 2003;17:245-58.

12. Qiu C, Xu W, Fratiglioni L. Vascular and psychosocial factors in Alzheimer's disease: epidemiological evidence toward intervention. $J$ Alzheimers Dis 2010;20:689-97.

13. Köhler S, Buntinx F, Palmer K, et al. Depression, vascular factors, and risk of dementia in primary care: a retrospective cohort study. $J$ Am Geriatr Soc 2015;63:692-8.

14. Poblador-Plou B, Calderón-Larrañaga A, Marta-Moreno J, et al. Comorbidity of dementia: a cross-sectional study of primary care older patients. BMC Psychiatry 2014;14:84.

15. Sahathevan R, Brodtmann A, Donnan GA. Dementia, stroke, and vascular risk factors; a review. Int J Stroke 2012;7:61-73.

16. Korczyn AD, Vakhapova V, Grinberg LT. Vascular dementia. J Neurol Sci 2012;322:2-10.

17. Popovic IM, Lovrencic-Huzjan A, Simundic AM, et al. Cognitive performance in asymptomatic patients with advanced carotid disease. Cogn Behav Neurol 2011;24:145-51.

18. Sztriha LK, Nemeth D, Sefcsik T, et al. Carotid stenosis and the cognitive function. J Neurol Sci 2009;283:36-40.

19. Perez FI, Gay JR, Taylor RL, et al. Patterns of memory performance in the neurologically impaired aged. Can J Neurol Sci 1975;2:347-55.

20. Koçer A, Koçer E, Beșir H, et al. Low scores on the Benton Facial Recognition Test associated with vertebrobasilar insufficiency. Med Hypotheses 2013;80:527-9.

21. Skoromets AA, Aliev KT, Lalayan TV, et al. Cognitive functions and treatment of their impairment in elderly patients with the vertebrobasilar insufficiency. Zhurnal Nevrol i Psikhiatrii Im SS Korsakova 2013;113:18-24

22. Stayman A, Nogueira RG, Gupta R. Diagnosis and management of vertebrobasilar insufficiency. Curr Treat Options Cardiovasc Med 2013;15:240-51

23. American Psychiatric Association. Diagnostic and Statistical Manual of Mental Disorders. Fourth Edition, 2000. 
24. Rossor MN, Fox NC, Mummery CJ, et al. The diagnosis of youngonset dementia. Lancet Neurol 2010;9:793-806.

25. Gucuyener DO, Yenilmez C, Ayranci U, et al. An analysis of changes in cerebral blood flood velocities in depressive pseudo-dementia and Alzheimer disease patients. Neurologist 2010;16:358-63.

26. Nishio $Y$, Hashimoto M, Ishii K, et al. Neuroanatomy of a neurobehavioral disturbance in the left anterior thalamic infarction. $J$ Neurol Neurosurg Psychiatry 2011;82:1195-200.

27. Yan $Y$, Liang L, Yuan $Y$, et al. Influence of stent-assisted angioplasty on cognitive function and affective disorder in elderly patients with symptomatic vertebrobasilar artery stenosis. Med Sci Monit 2014;20:1129-36.

28. Mathiesen EB, Waterloo K, Joakimsen O, et al. Reduced neuropsychological test performance in asymptomatic carotid stenosis: the Tromsø Study. Neurology 2004;62:695-701.

29. Moftakhar R, Turk AS, Niemann DB, et al. Effects of carotid or vertebrobasilar stent placement on cerebral perfusion and cognition. AJNR Am J Neuroradiol 2005;26:1772-80.

30. Moroney JT, Bagiella E, Desmond DW, et al. Risk factors for incident dementia after stroke. Role of hypoxic and ischemic disorders. Stroke 1996;27:1283-9.

31. Prins ND, van Dijk EJ, den Heijer T, et al. Cerebral small-vessel disease and decline in information processing speed, executive function and memory. Brain 2005;128:2034-41.

32. Elias MF, Sullivan LM, D'Agostino RB, et al. Framingham stroke risk profile and lowered cognitive performance. Stroke 2004;35:404-9.

33. Breteler MMB. Vascular risk factors for Alzheimer's disease. Neurobiol Aging 2000;21:153-60.
34. Kivipelto M, Ngandu T, Laatikainen T, et al. Risk score for the prediction of dementia risk in 20 years among middle aged people: a longitudinal, population-based study. Lancet Neurol 2006;5:735-41.

35. Jorm AF, Jolley D. The incidence of dementia: a meta-analysis. Neurology 1998;51:728-33.

36. Guilbert JJ. The World Health Report 2002-reducing risks, promoting healthy life. Educ Health 2003;16:230.

37. Park KC, Yoon SS, Rhee HY. Executive dysfunction associated with stroke in the posterior cerebral artery territory. J Clin Neurosci 2011;18:203-8

38. Mathew NT, Meyer JS. Pathogenesis and natural history of transient global amnesia. Stroke 1974;5:303-11.

39. Nishio K, Ihara M, Yamasaki N, et al. A mouse model characterizing features of vascular dementia with hippocampal atrophy. Stroke 2010;41:1278-84.

40. Jiwa NS, Garrard P, Hainsworth AH. Experimental models of vascular dementia and vascular cognitive impairment: a systematic review. $J$ Neurochem 2010;115:814-28.

41. Wilcock DM, Colton CA. Immunotherapy, vascular pathology, and microhemorrhages in transgenic mice. CNS Neurol Disord Drug Targets 2009;8:50-64.

42. Lin CJ, Tu PC, Chern CM, et al. Connectivity features for identifying cognitive impairment in presymptomatic carotid stenosis. PLoS One 2014;9:e85441.

43. Cheng HL, Lin CJ, Soong BW, et al. Impairments in cognitive function and brain connectivity in severe asymptomatic carotid stenosis. Stroke 2012;43:2567-73. 